\section{Schmerzfrei für 90 Tage}

\section{PERIPHERE NEUROPATHISCHE SCHMERZEN Die topische Behandlung mit hochdosiertem Capsaicin lindert bei einmaliger Anwendung neuro- pathische Schmerzen über 90 Tage.}

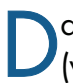
as Capsaicin 8\%-Pflaster Qutenza ${ }^{\circledR}$ vermutlich ab Oktober 2010 verfügbar) wird für 30 bis 60 Minuten direkt auf das schmerzende Areal geklebt. Es setz† dort den hochselektiven TRPV1-RezeptorAgonisten (Transient Receptor Potential Vanilloid) Capsaicin frei, der unmittelbar auf die für die Schmerzübertragung und -modulation verantwortlichen Nozizeptoren wirkt. Bei peripheren neuropathischen Schmerzen kommt es nach einer einzigen Anwendung zu einer raschen Analgesie, die 90 Tage anhält, berichtete Prof Dr. Thomas Tölle, München.

Effektivität und Verträglichkeit sind in 13 klinischen Studien mit fast 2400 dokumentiert. So reduzierte das Pflaster über 60 Minuten angewendet bei $44 \%$ von 402 Patienten mit postherpetischer Neuralgie die Schmerzen um mindestens $30 \%$ verglichen mit aktivem Plazebo, das 0,04\% des Wirkstoffs enthielt [1]. Die Wirkung setzte am zweiten Tag ein, hielt über 90 Tage und wurde in Monotherapie oder in Kombination mit anderen Medikamenten erzielt. Gleiche Ergebnisse liefert eine Studie mit $307 \mathrm{~Pa}-$ tienten mit HIV-assoziierter Neuralgie [2]. Hier kam es bei $38 \%$ schon nach 30 -minütiger Tragdaver zu der relevanten Schmerzreduktion von $30 \%$, so Tölle.

Auch bei wiederholter Anwendung war das Pflaster unverändert wirksam und gut verträglich. Michael Koczorek

Backonja M et al. Lancet Neurology 2008; 7 (12): 1106-1112 2 Simpson DM et al. Neurology 2008; 70(24): 2305-2313 Pressekonferenz „Neuartige Schmerztherapie mit hochdosiertem Capsaicin-Pflaster $8 \%$ bei peripheren neuropathischen Schmerzen", Frankfurt/Main, 19. März 2010; Veranstalter: Astellas Pharma GmbH

\title{
Schutz vor opioidinduzierter Hyperalgesie
}

\section{OPIOIDBASISTHERAPIE Wenn der Opioidspiegel immer wieder rasch abfällt, droht eine synaptische Langzeitpotenzierung an C-Fasern mit anhaltender Verstärkung der Nozizeption. Davor schützł ein gleich- mäßiges Plateau durch Gabe eines rund um die Uhr wirksamen Opioids.}

Selbst bei zusätzlicher Gabe kurz wirkSsamer Opioide gegen Durchbruchschmerzen, etwa bei Umlagerung oder Verbandwechsel, werde durch die gleichmäßige Basistherapie ein Opioidabfall "ins Bodenlose" verhindert, erklärte Prof. Dr. Walter Zieglgänsberger, München.

Zur Frage, welche Kurzzeittherapie als Bolus zum retardierten Opioid geeignet sei, erklärte Dr. Johannes Horlemann aus Kevelaer bei Wesel: „Bei Schmerzspitzen von etwa 30 bis 60 Minuten eignet sich kurz wirksames Fentanyl, bei nächtlichen ,Schmerzbuckeln' von drei bis vier Stunden eher ein Morphin und bei neuropathischen Schmerzen ein Antiepileptikum." Für die Basistherapie mit einem lang wirksamen Opioid bevorzugt Horlemann Jurnista ${ }^{\circledR}$ : „Das ist Hydromorphon in einer ganz speziellen Darreichungsform, die eine gleichmäßige Wirkstoffabgabe über 24 Stunden garantiert. Die Halbwertdauer liegt bei 27 bis 29 Stunden, das ist deutlich länger als bei den anderen Opioidpräparaten."

Simone Reisdorf

Veranstaltung „Opioid-Therapie: mit gleichmäßigem Plasmaspiegel gegen Hyperalgesie und Schmerzchronifizierung" im Rahmen des Schmerz- und Palliativtags, Frankfurt/Main,

18. März 2010; Veranstalter: Janssen-Cilag

\section{Schnelle Schmerzlinderung} schon nach 10 Minuten

Tumorbedingte Durchbruchschmerzen (DBS) sind eine Herausforderung bei der Betreuung von Krebspatienten. Meistens sind sie nach 30 Minuten schon wieder vorbei - zU spät für eine Behandlung mit oralen nicht retardierten Morphinpräparaten.

Eine Studie untersuchte nun Wirksamkeit und Langzeitverträglichkeit von oral auflösenden sublingualen Fentanyltabletten. Das primäre Studienziel bestand darin, die Summe der Schmerzintensitätsdifferenz (Sum of Pain Intensity Difference, SPID) über 30 Minuten nach Tabletteneinnahme festzustellen, mit einem ersten Messpunkt nach zehn Minuten. Die sekundären Studienziele betrafen die Schmerzintensitätsdifferenz (Pain Intensity Difference, PID) sowie die Schmerzlinderung (Pain Relief, PR) über alle Messzeitpunkte innerhalb von 60 Minuten nach Tabletteneinnahme.

Insgesamt konnten die Daten von 64 Patienten ausgewertet werden. Dabei zeigte sich mit Abstral ${ }^{\circledR}$ eine signifikante Verbesserung der SPID30. Zudem war schon vom ersten Messpunkt an - also nach nur zehn Minuten - eine Verbesserung von PID und PR zu erkennen [1].

Insgesamt wurde die sublinguale Fentanyltablette sowohl systemisch als auch lokal gut vertragen. Zu den am häufigsten aufgetretenen Nebenwirkungen zählten - typisch für Opioide - Nausea, Erbrechen und Somnolenz [1]. Aufgrund der neuartigen patentierten Galenik werden bei Kontakt mit Speichel bioadhäsive Partikel mit mikronisiertem Fentanyl freigesetzt. So kann sich die Tablette unter der Zunge in zehn bis 15 Sekunden auflösen. Da mechanische Belastung und Reizung der Mukosa so gut wie ausgeschlossen sind, eignet sich Abstral ${ }^{\circledR}$ auch gut für Patienten mit Mukositis/ Mundtrockenheit, die häufig nach einer Chemo- oder Radiotherapie auftritt.

Nach Informationen der Arzneimittel ProStrakan GmbH

Rauck RL, Tark M, Reyes E, Hayes TG, Bartkowiak AJ, Hassman D, Nalamachu S, Derrick R, Howell J. Efficacy and long-term tolerability of sublingual fentanyl orally disintegrating tablet in the treatment of breakthrough cancer pain. Curr Med Res Opin 2009. 25 (12): 2877-2885 